\title{
Marital status and survival in patients with rectal cancer
}

\section{A population-based STROBE cohort study}

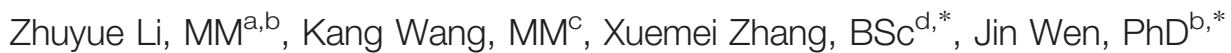

\begin{abstract}
To examine the impact of marital status on overall survival (OS) and rectal cancer-specific survival (RCSS) for aged patients.

We used the Surveillance, Epidemiology and End Results database to identify aged patients (>65 years) with early stage rectal cancer (RC) (T1-T4, NO, MO) in the United States from 2004 to 2010. Propensity score matching was conducted to avoid potential confounding factors with ratio at 1:1. We used Kaplan-Meier to compare OS and RCSS between the married patients and the unmarried, respectively. We used cox proportion hazard regressions to obtain hazard rates for OS, and proportional subdistribution hazard model was performed to calculate hazard rates for RCSS.

Totally, 5196 patients were included. The married (2598 [50\%]) aged patients had better crude 5-year overall survival rate (64.2\% vs $57.3 \%, P<.001)$ and higher crude 5 -year cancer-specific survival rate $(80 \%$ vs $75.9 \%, P<.001)$ than the unmarried $(2598(50 \%))$, respectively. In multivariate analyses, married patients had significantly lower overall death than unmarried patients $(\mathrm{HR}=0.77,95 \%$ $\mathrm{Cl}=0.71-0.83, P<.001)$, while aged married patients had no cancer-specific survival benefit versus the unmarried aged patients $(\mathrm{HR}=0.92,95 \% \mathrm{Cl}=0.81-1.04, P=.17)$.

Among old population, married patients with early stage $\mathrm{RC}$ had better OS than the unmarried, while current evidence showed that marital status might have no protective effect on cancer-specific survival.
\end{abstract}

Abbreviations: $\mathrm{Cl}=$ confidence interval, $\mathrm{HR}=$ hazard rate, $\mathrm{OS}=$ overall survival, $\mathrm{RC}=$ rectal cancer, $\mathrm{RCSS}=$ rectal cancerspecific survival, SEER = Surveillance, Epidemiology, and End Results, VIF = variance inflation factor.

Keywords: marital status, propensity score-matched analysis, rectal cancer, SEER, survival

\section{Introduction}

Rectal cancer (RC) leads to heavy disease burden and tremendous medical costs worldwide. As the third most commonly cancer, 135,430 colorectal cancer cases were newly diagnosis and 50,260 patients died of colorectal cancer in 2017 in United States. ${ }^{[1]}$ Researchers have studied some pathological factors such as gene expression $^{[2]}$ and reaction of chemotherapy ${ }^{[3,4]}$ that might affect survival of RC, however, it is possible for sociodemographic

Editor: Massimo Tusconi.

The authors have no conflicts of interest to disclose.

Supplemental Digital Content is available for this article.

a West China Hospital/West China School of Nursing, ${ }^{b}$ Institute of Hospital Management, West China Hospital, Sichuan University, China, ${ }^{\circ}$ Department of the Endocrine and Breast Surgery, The First Affiliated Hospital of Chongqing Medical University, Chongqing Medical University, Chongqing, ${ }^{d}$ Center of Gerontology and Geriatrics, West China Hospital, Sichuan University, Chengdu, China.

* Correspondence: Xuemei Zhang, Center of Gerontology and Geriatrics, West China Hospital, Guo Xue Xiang 37, Chengdu, PRC, 610041

(e-mail: zxm0709@126.com); Jin Wen, Institute of Hospital Management, West China Hospital, Guo Xue Xiang 37, Chengdu, PRC, 610041

(e-mail: huaxiwenjin@163.com).

Copyright (๑) 2018 the Author(s). Published by Wolters Kluwer Health, Inc. This is an open access article distributed under the Creative Commons Attribution-NoDerivatives License 4.0, which allows for redistribution, commercial and non-commercial, as long as it is passed along unchanged and in whole, with credit to the author.

Medicine (2018) 97:18(e0637)

Received: 26 November 2017 / Accepted: 12 April 2018

http://dx.doi.org/10.1097/MD.0000000000010637 factors to affect survival of patients with RC. Marital status is known as one type of social support with beneficial psychological properties. Previous studies have reported that marital status provide protective effect for some diseases, ${ }^{[5-9]}$ for instance, married patients generally obtained early detection, be more likely to receive surgical treatment, and experienced long-term survival. ${ }^{[10-12]}$ However, the relation between marital status and prognosis of RC remains unknown, particularly with respect to the older group who are easy to expose to poor social support.

Using of treatments such as low anterior resection surgery, chemotherapy, and radiotherapy has become more and more popular. However, those treatments could result in enormous impact on patients' life such as reduction of quality of life and incidence of complications. One of reasons contributing to decreased quality of life can be the presence of ostomies which are mostly used for minimize possible complications of an anastomotic leakage. Ostomies generate significant difficulties for patients both physically and psychologically. ${ }^{[13-21]}$ Thus, the effect of social support should be emphasized. Early study found significant association between colon cancer and marital status $^{[12]}$ while they did not discuss effect on RC that is closely related but distinct to colon cancer. However, the effect of marriage on different cancer was inconsistent, some studies revealed protective effect, ${ }^{[8,10,12,22-24]}$ some researchers found $\operatorname{mix}$ effect ${ }^{[25-27]}$ and Jatoi et $\mathrm{al}^{[28]}$ reported no significant relationship. Furthermore, previous studies had significant imbalance baseline that married people were more likely to be diagnosed at an earlier stage compared to the unmarried. ${ }^{[9,12]}$

To best of our knowledge, little research focused on the association between RC and marital status among the elderly 
population. Based on the National Cancer Institute's Surveillance, Epidemiology, and End Results (SEER) program, the purpose of this study is to explore the relationship between marital status and survival in patients with RC.

\section{Methods}

\subsection{Study design and data source}

A population-based historical cohort study was designed. The institutional review board of West China Hospital approved the study. We acquired the data from SEER database (http://seer. cancer.gov/) which is a national cancer database sponsored by the National Cancer Institute, and we retrospective research relationship of marital status on survival of RC among old patients. Data of SEER database represented about 30\% proportion of American due to it covered 18 population-based registries. SEER dataset includes demographic information of patients, cancer diagnosis, and treatment information, as well as cause-of-death information.

\subsection{Identification of patients}

Patients with RC were identified according to the following inclusion criteria: The codes of primary cancer site were 199 and 209 which were identified by International Classification of Diseases for Oncology (ICD-O-3). ${ }^{[29]}$ We defined the histological subtype as adenocarcinoma that identified with ICD-O-3 codes 814,821 , and 822. Stage I or stage II (T1-T4, N0, M0) patients. Patients whose age over 65 years and diagnosed in 2004 to 2010 were chosen. Patients without adequate information on marital status, race, grade, radiotherapy, surgery type, and survival time were excluded.

\subsection{Identification of key variables}

We extracted relevant information by the SEER *Stat software (version 8.3.4), including marital status, year of diagnosis, age,

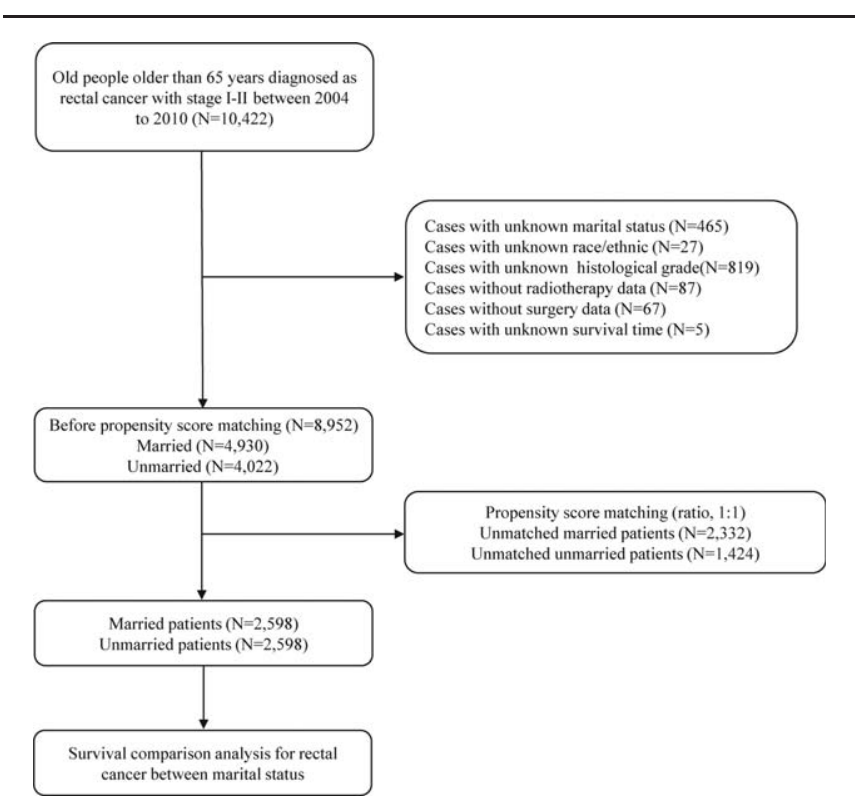

Figure 1. Flow chart of data processing and included patients' identification.

race, sex, grade, American Joint Committee on Cancer (AJCC) 6th tumor node metastasis (TNM) staging classification, radiotherapy, chemotherapy, surgery type, survival time (in months), vital status, cause-specific death classification, and other cause of death classification. Surgery types were defined by SEER codes as local (10-28), radical (30-80), and nonsurgery $(0)$. We defined marital status as married (including cohabit as well as married) and single (never married, divorced, separated or widowed). Endpoints were defined as overall survival (OS) which was measured as an internal from time of diagnosis to death or date of last contact (December 2014). Rectal cancer-specific survival (RCSS) was estimated as time
Raw Treated

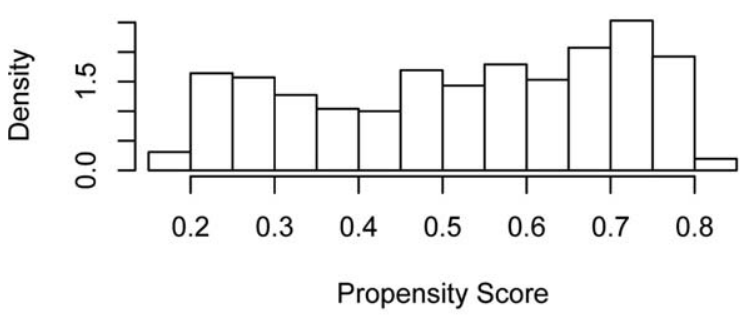

Raw Control

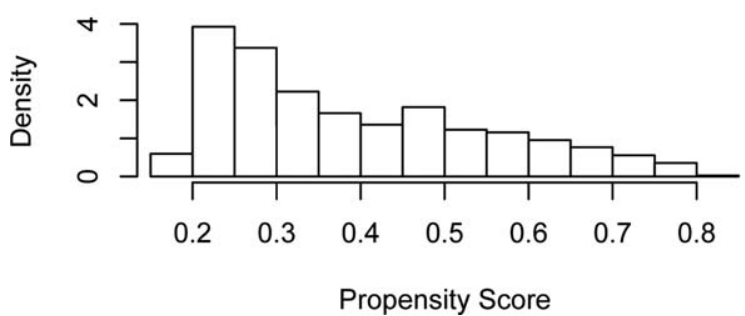

Matched Treated
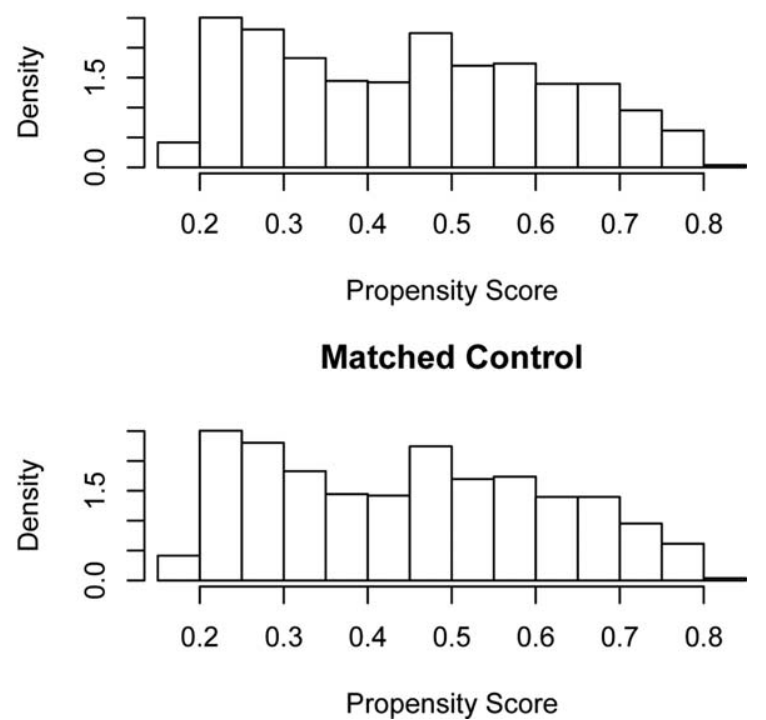

Figure 2. Characteristic of included patients' baseline before and after propensity score matching. 
from RC diagnosis to death from RC or data of last contact (December 2014).

\subsection{Propensity score matching}

We carried out an inverse probability propensity score weighting $^{[30]}$ to balance baseline using "MatchIt" $\mathrm{R}$ package. ${ }^{[31]}$ Data of year of diagnosis, age of patients, race, $\mathrm{T}$ stages based on 6th AJCC, radiotherapy, chemotherapy, and surgery type were used to merge propensity scores for individuals through a logistic regression model, and balanced groups had a ratio at 1:1.

\subsection{Statistical methods}

Demographic information of patients, tumor, and treatment characteristics were compared between married and single groups using Pearson Chi-square test for categorical variables. Log-rank tests and Cox proportion hazard regressions that matched by propensity scores were used to compare the differences between married and single in OS, and we calculated hazard ratios (HR) with $95 \%$ confidence interval (CI). Specifically, we conducted proportional subdistribution hazard model by "cmprsk" R package ${ }^{[32]}$ to obtain HRs with $95 \% \mathrm{CI}$ for RCSS. To avoid influence of multicollinearity between variables that be highly related, we only included one of them into final model (e.g., AJCC stage and AJCC T stage). VIF was used for multicollinearity diagnostics by "rms" package in R. All the adjusted HRs were acquired when fitting multivariable models, which involved to adjust for the variables in propensity score matching procedure. In addition, in this study, interaction analyses were performed to assess the difference of survival

\section{Table 1}

Baseline characteristics of aged patients with early stage (stage I/II) rectal cancer included in this study $(\mathrm{N}=5196)$.

\begin{tabular}{|c|c|c|c|c|c|c|}
\hline \multirow[b]{2}{*}{ Characteristic } & \multicolumn{2}{|c|}{$\begin{array}{c}\text { Unmatched } \\
\text { No. of patients (\%) }\end{array}$} & \multirow[b]{2}{*}{$P$} & \multicolumn{2}{|c|}{$\begin{array}{c}\text { Matched } \\
\text { No. of patients (\%) }\end{array}$} & \multirow[b]{2}{*}{$P$} \\
\hline & Married ${ }^{*}$ (2598) & Single $^{\dagger}$ (2598) & & Married ${ }^{*}$ (2598) & Single $^{\dagger}$ (2598) & \\
\hline 2004-2006 & 2224 (45.1) & $1847(45.9)$ & .74 & 1219 (46.9) & $1172(45.1)$ & .33 \\
\hline 2007-2008 & $1385(28.1)$ & $1118(27.8)$ & & $697(26.8)$ & $701(27.0)$ & \\
\hline 2009-2010 & $1321(26.8)$ & 1057 (26.3) & & $682(26.3)$ & $725(27.9)$ & \\
\hline \multicolumn{7}{|l|}{ Age, years } \\
\hline $75-79$ & 1097 (22.3) & $846(21.0)$ & & 609 (23.4) & 605 (23.3) & \\
\hline $80-84$ & $720(14.6)$ & $843(21.0)$ & & $466(17.9)$ & $424(16.3)$ & \\
\hline$\geq 85$ & $368(7.5)$ & $817(20.3)$ & & $250(9.6)$ & $289(11.1)$ & \\
\hline \multicolumn{7}{|l|}{ Sex } \\
\hline Male & 3416 (69.3) & 1509 (37.5) & $<.001$ & $1396(53.7)$ & 1389 (53.5) & .85 \\
\hline Female & $1514(30.7)$ & $2513(62.5)$ & & 1202 (46.3) & 1209 (46.5) & \\
\hline \multicolumn{7}{|l|}{ Grade } \\
\hline I & $486(9.9)$ & 357 (8.9) & .08 & 227 (8.7) & $234(9.0)$ & .92 \\
\hline$\|$ & 3961 (80.3) & 3223 (80.1) & & 2146 (82.6) & 2135 (82.2) & \\
\hline III/IV & $464(9.4)$ & 417 (10.4) & & $225(8.7)$ & $229(8.8)$ & \\
\hline \multicolumn{7}{|l|}{ AJCC stage } \\
\hline I & 2583 (52.4) & 1980 (49.2) & .003 & 1297 (49.9) & 1285 (49.5) & .74 \\
\hline$\|$ & 2347 (47.6) & 2042 (50.8) & & $1301(50.1)$ & $1313(50.5)$ & \\
\hline \multicolumn{7}{|l|}{ AJCC T stage } \\
\hline $\mathrm{T} 1$ & 1278 (25.9) & 968 (24.1) & $<.001$ & $636(24.5)$ & 605 (23.3) & .52 \\
\hline T2 & 1305 (26.5) & 1012 (25.2) & & 661 (25.4) & 680 (26.2) & \\
\hline T3 & 2102 (42.6) & 1760 (43.8) & & $1160(44.6)$ & $1153(44.4)$ & \\
\hline T4 & $245(5.0)$ & $282(7.0)$ & & $141(5.4)$ & $160(6.2)$ & \\
\hline \multicolumn{7}{|l|}{ Chemotherapy } \\
\hline No/Unknown & 3048 (61.8) & 2760 (68.6) & $<.001$ & $1712(65.9)$ & 1725 (66.4) & .70 \\
\hline Yes & 1882 (38.2) & 1262 (31.4) & & 886 (34.1) & 873 (33.6) & \\
\hline
\end{tabular}

AJCC $=$ American Joint Committee on Cancer system

*Married patients including common law.

† Unmarried status including never married, divorced, separated and widowed.

\$Included American Indian/AK Native, Asian/Pacific Islander. 

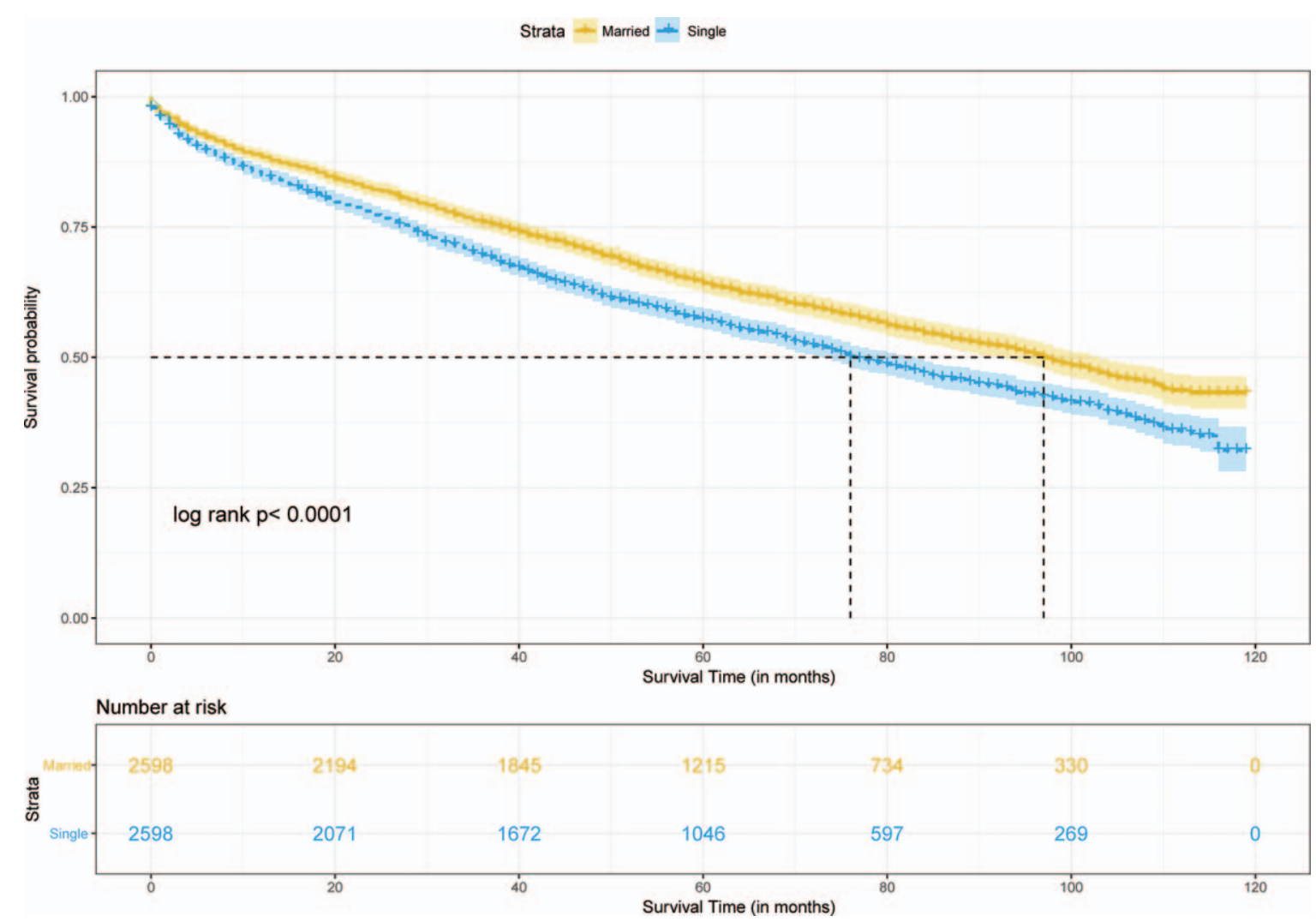

Figure 3. Survival curve of overall survival on aged rectal cancer patients.
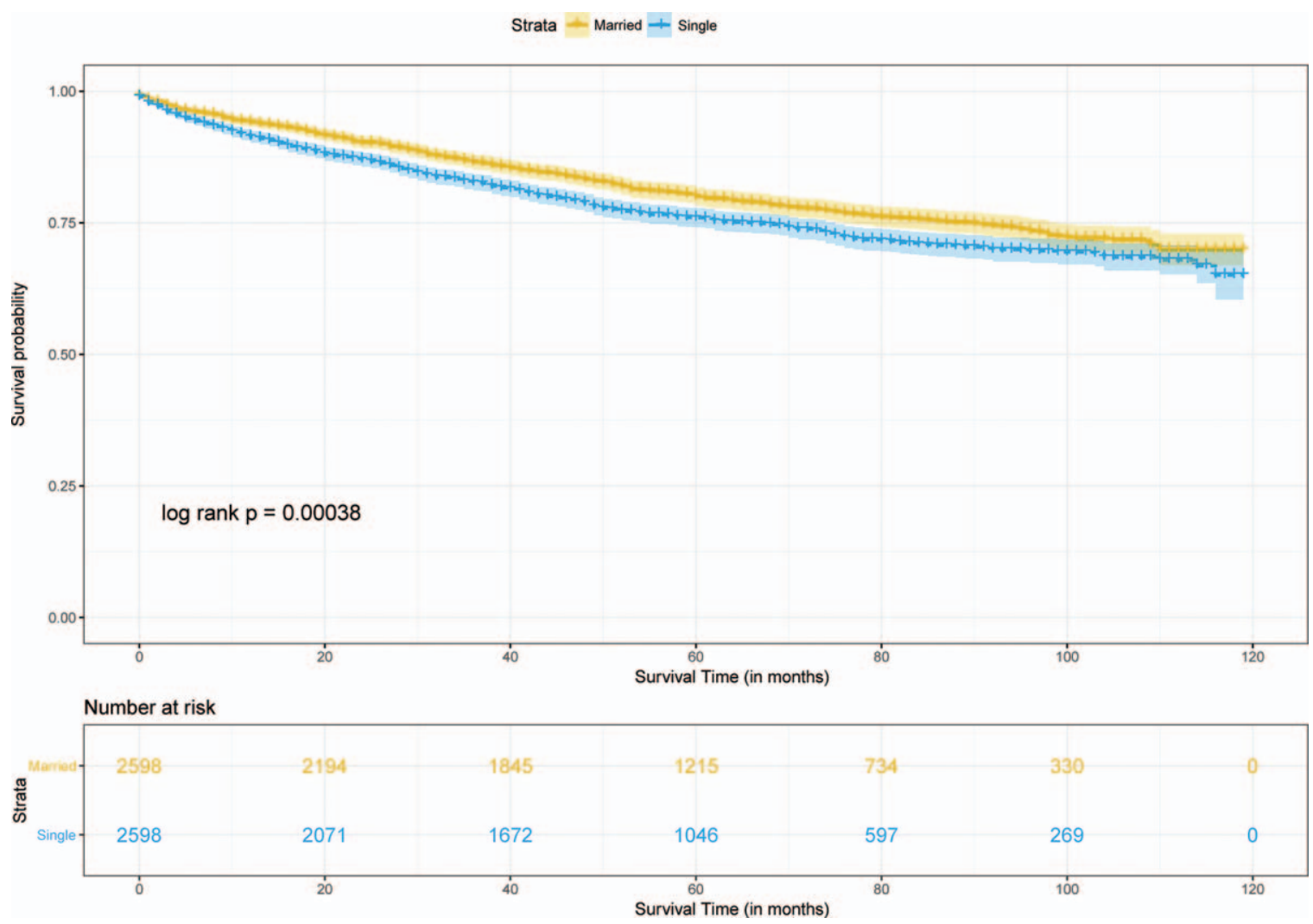

Figure 4. Survival curve of cancer-specific survival on aged rectal cancer patients. 

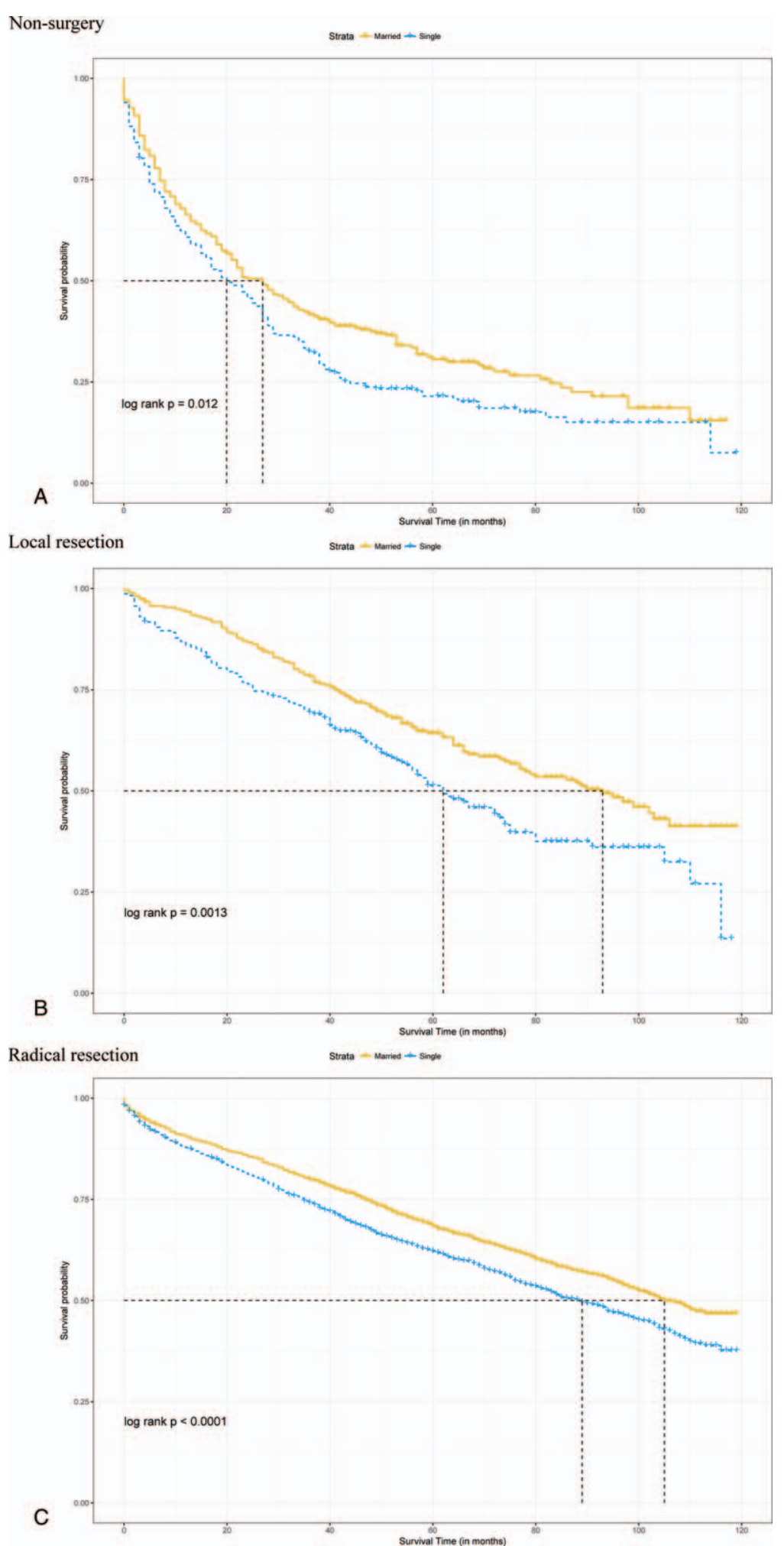

Figure 5. Overall survival with $95 \%$ Cls for rectal cancer patients. (A) Overall survival for rectal cancer patients with nonsurgery. (B) Overall survival for rectal cancer patients with local resection. (C) Overall survival for rectal cancer patients with radical resection. $\mathrm{Cl}=$ confidence intervals.

conferred by marital status across potential modifiers. Stratified survival analyses were conducted on particularly different groups.
All $P$ values were calculated from 2 -sided tests with threshold of .05 to evaluate statistical significance, and all statistical analyses were performed by $\mathrm{R}$ software (version 3.4.3). 


\section{Results}

\subsection{Participants and characteristic}

According to SEER database, from January 1st, 2004 to December 31st, 2010, 29,144 elderly patients ( $\geq 65$ years) were diagnosed with rectal cancer. Among these patients, we identified 10,422 cases that were confirmed with adenocarcinoma and pathological stage I to II. According to inclusion criteria, we enrolled 8952 patients before propensity score matching. Finally, 5196 rectal cancer aged patients were enrolled in our study after propensity score matching, 2598 aged married patients and 2598 aged single patients, respectively. Figure 1 displayed the flow of data processing and patients' identification. Figure 2 described results of propensity score matching by histograms. Characteristics of baseline in the present study were showed in Table 1. Before propensity score matching, age at diagnosis, gender, race, AJCC T stage, surgery type, radiotherapy, and chemotherapy were significantly different between married RC patients and those single. In the matched groups, most patients were white in both groups ( $86.5 \%$ in married group; $82.8 \%$ in single group), whereas the composition of other races varies. Married RC patients had less black $(4.6 \%)$ and more other races $(8.9 \%)$ including American Indian/AK Native, Asian/Pacific Islander. Unmarried group had more black $(9.6 \%)$ and less other race $(7.5 \%)$. Except for race, the baseline of groups was similar and comparable indicating that no significant difference between the matched groups existed regarding to potential prognostic factors.

\subsection{Outcomes of overall survival and rectal cancer specific survival}

Median follow-up durations for the married and unmarried cohorts were 4.75 years and 4.17 years, respectively, with maximum of 9.92 years for both groups. Data showed that 1107 married patients $(42.6 \%)$ dead at the end of follow-up versus 1284 unmarried patients $(49.4 \%)$. Crude 5 -year OS rate was $64.2 \%$ in the matched married group and $57.3 \%$ in the matched unmarried group (log-rank test, $\mathrm{HR}=0.79,95 \% \mathrm{CI}=0.73-0.85$, $P<.001$, Fig. 3). A total of $529(20.4 \%)$ matched married patients were died of RC and $603(23.2 \%)$ matched unmarried patients were died of RC. Crude 5 -year RCSS rate was $80 \%$ in the matched married group and $75.9 \%$ in the matched unmarried group (log-rank test, $\mathrm{HR}=0.81,95 \% \mathrm{CI}=0.72-0.91, P<.001$, Fig. 4). By looking on the hazard ratios particularly different treatment groups (chemotherapy, radiotherapy, surgery), we stratified the survival analyses in these subset of groups, which displayed in Figs. 5 and 6. Same survival benefit on married RC patients was observed in all the subgroups, whereas different surgery subsets showed different median survival time in both groups.

Adjusted HR for OS between married patients and the unmarried was revealed by inverse propensity score matchedmultivariable Cox proportional hazards models with sex, age, year of diagnosis, race, stage, grade, chemotherapy, radiotherapy, surgery type $(\mathrm{HR}=0.77,95 \% \mathrm{CI}=0.71-0.83, P<.001$,
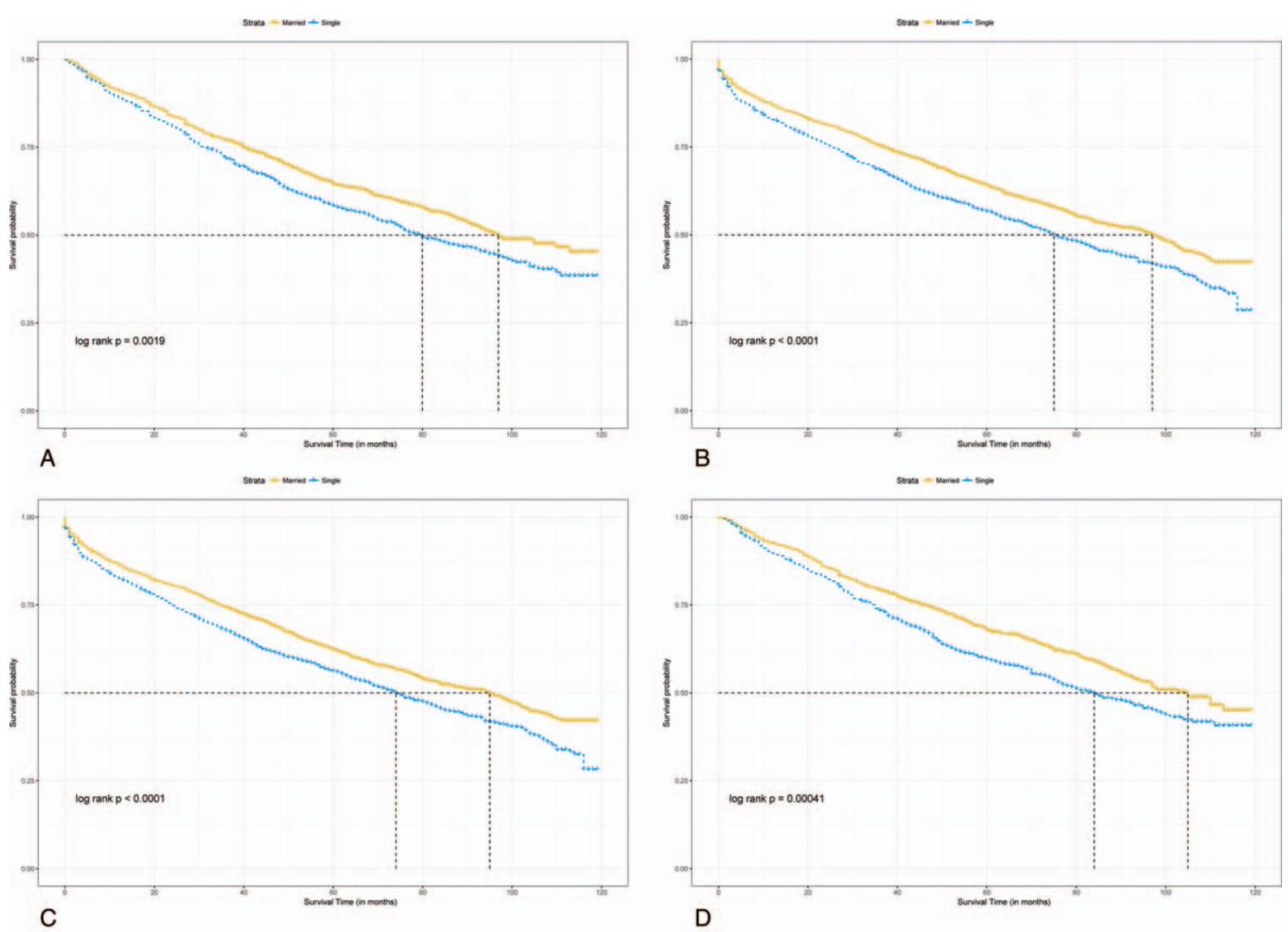

Figure 6. Overall survival with 95\% Cls for rectal cancer patients. (A) Overall survival for rectal cancer patients with nonradiotherapy. (B) Overall survival for rectal cancer patients with radiotherapy. (C) Overall survival for rectal cancer patients with nonchemotherapy. (D) Overall survival for rectal cancer patients with chemotherapy. $\mathrm{Cl}=$ confidence intervals. 
Table 2

Multivariable analysis of marital status for overall survival and cancer-specific survival in the unmatched cohort.

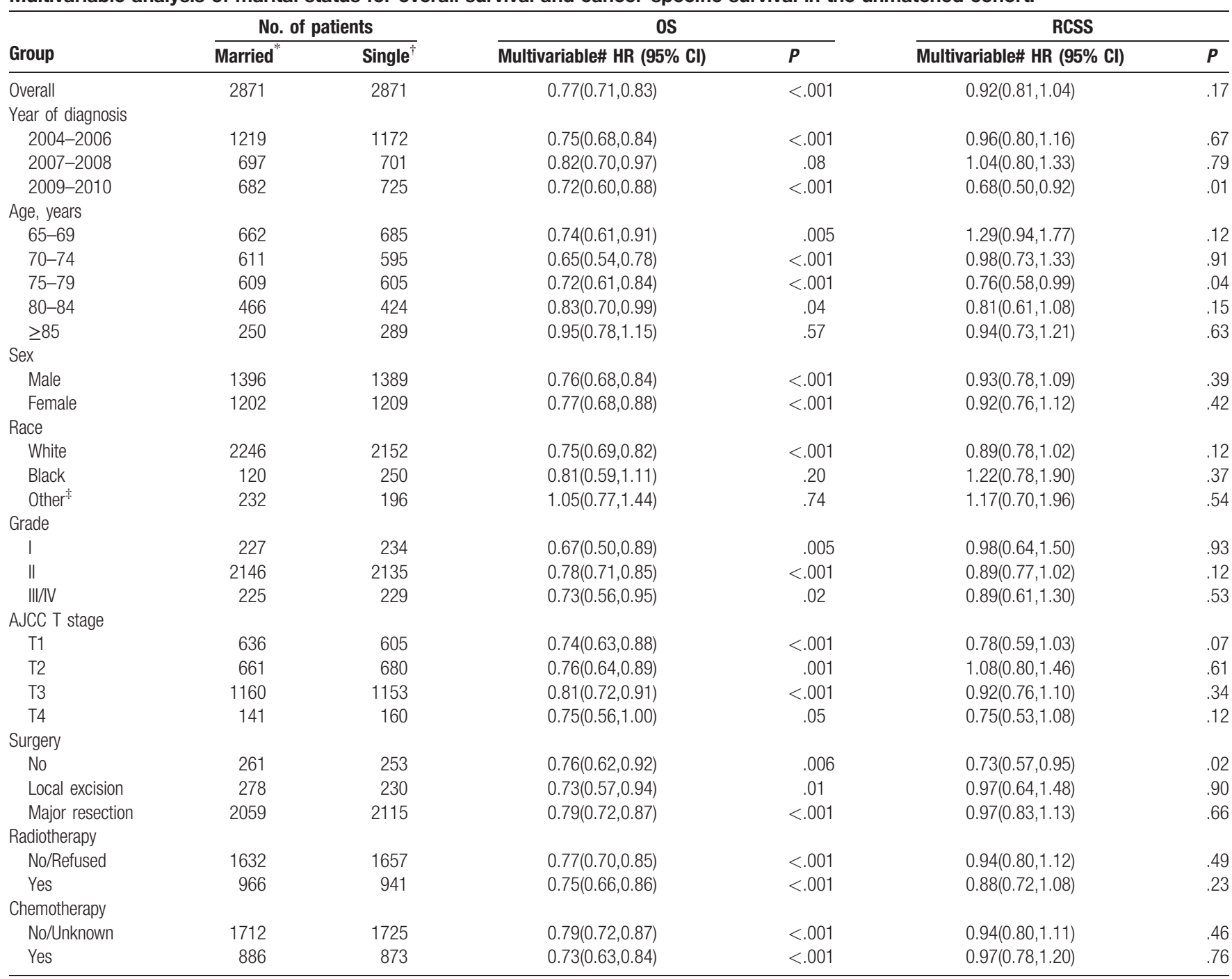

AJCC $=$ American Joint Committee on Cancer system, $\mathrm{Cl}=$ confidence interval, $\mathrm{HR}=$ hazard ratio, $\mathrm{OS}=$ overall survival, $\mathrm{RCSS}=$ rectal cancer specific survival.

* Married patients including common law.

† Unmarried status including never married, divorced, separated and widowed.

\# Adjusting factors included sex, age, year of diagnosis, race, stage, grade, chemotherapy, radiotherapy, and surgery type.

*Included American Indian/AK Native, Asian/Pacific Islander.

Table 2). After adjusting multiple confounding factors, RCSS was similar between the matched groups (HR: 0.92, 95\% CI $=0.81-$ $1.04, P=.17$, Table 2). According to the multicollinearity diagnostic (VIF) result which showed on Supplementary table 1 on line, http://links.lww.com/MD/C231, there is no collinearity between the variables in the regression model.

Table 2 illustrated the results of interaction analyses. According to the results of interaction analyses, OS advantage among married patients versus unmarried patients disappeared in subgroup with age $>85$ years (HR: $0.95,95 \% \mathrm{CI}=0.78-1.15$, $P=.57$ ) and race of black or other (American Indian/AK Native, Asian/Pacific Islander) (HR: 0.81, 95\% CI: 0.59-1.11, $P=.20$; HR: $1.05,95 \%$ CI: $0.77-1.44, P=.74$, respectively). Among interaction analyses regarding to RCSS, we found that patients diagnosed in 2009 to 2010 (HR: 0.68, 95\% CI: 0.50-0.92, $P=.01$ ), patients aged at 75 to 79 years (HR: $0.76,95 \%$ CI: $0.58-$ $0.99, P=.04)$ and patients without surgery (HR: $0.73,95 \% \mathrm{CI}$ : $0.57-0.95, P=.02)$ had significant difference on cancer-specific survival between different marital status. Multivariate Cox analyses confirmed the independent prognostic significance of year of diagnosis, age at diagnosis, gender, race, grade, AJCC T stage, surgery type, and chemotherapy. Radiotherapy did not reach significance with this test. The whole model is shown in Table 3. Interestingly, inverted hazard ratios were found of the chemotherapy category in RCSS (univariate analysis: HR: 1.15, 95\% CI: $1.02-1.30, P=.02$; Multivariate analysis: HR: 0.77 , 95\% CI: $0.63-0.95, P=.01)$.

\section{Discussion}

In the retrospective longitudinal cohort study, we found that married aged patients experience a significant benefit of OS than the unmarried. The association between marital status and OS remained after adjusting for demographics, tumor characteristics, and treatment and we observed a relative reduction in overall death at $23 \%$. Interestingly, the protective effect of 
Hazard ratios for overall survival and for cancer-specific survival.

\begin{tabular}{|c|c|c|c|c|c|c|c|c|}
\hline \multirow[b]{3}{*}{ Group } & \multicolumn{4}{|c|}{ Overall survival } & \multicolumn{4}{|c|}{ Rectal cancer-specific survival } \\
\hline & \multicolumn{2}{|c|}{ Univariate analysis $^{*}$} & \multicolumn{2}{|c|}{ Multivariate analysis $^{\dagger}$} & \multicolumn{2}{|c|}{ Univariate analysis $^{*}$} & \multicolumn{2}{|c|}{ Multivariate analysis $^{\dagger}$} \\
\hline & HR (95\% Cl) & $P$ & HR (95\% Cl) & $P$ & HR (95\% Cl) & $P$ & HR (95\% Cl) & $P$ \\
\hline 2004-2006 & Reference & & Reference & & Reference & & & \\
\hline 2007-2008 & $0.90(0.82,1.00)$ & .04 & $0.86(0.78,0.95)$ & .004 & $1.00(0.87,1.14)$ & .96 & & \\
\hline $2009-2010$ & $0.87(0.77,0.97)$ & .01 & $0.82(0.73,0.92)$ & $<.001$ & $0.87(0.74,1.02)$ & .08 & & \\
\hline $70-74$ & $1.48(1.29,1.70)$ & $<.001$ & $1.50(1.31,1.72)$ & $<.001$ & $1.33(1.10,1.60)$ & .003 & $1.37(1.13,1.65)$ & .001 \\
\hline $75-79$ & $1.98(1.74,2.25)$ & $<.001$ & $2.00(1.75,2.27)$ & $<.001$ & $1.51(1.26,1.82)$ & $<.001$ & $1.56(1.30,1.88)$ & $<.001$ \\
\hline $80-84$ & $2.70(2.37,3.09)$ & $<.001$ & $2.65(2.32,3.03)$ & $<.001$ & $2.09(1.73,2.52)$ & $<.001$ & $2.09(1.73,2.53)$ & $<.001$ \\
\hline$\geq 85$ & $5.16(4.49,5.93)$ & $<.001$ & $4.35(3.77,5.03)$ & $<.001$ & $4.28(3.52,5.20)$ & $<.001$ & $3.60(2.95,4.40)$ & $<.001$ \\
\hline \multicolumn{9}{|c|}{ 管 } \\
\hline Male & Reference & & Reference & & Reference & & Reference & \\
\hline \multicolumn{9}{|l|}{ Grade } \\
\hline I & Reference & & Reference & & Reference & & Reference & \\
\hline$\|$ & $1.05(0.91,1.22)$ & .47 & $1.03(0.89,1.19)$ & .74 & $1.12(0.90,1.40)$ & .30 & $1.10(0.87,1.35)$ & .45 \\
\hline III/IV & $1.31(1.08,1.58)$ & .005 & $1.21(1.00,1.46)$ & .047 & $1.62(1.24,2.13)$ & $<.001$ & $1.49(1.14,1.96)$ & .004 \\
\hline \multicolumn{9}{|l|}{ AJCC T stage } \\
\hline $\mathrm{T} 1$ & Reference & & Reference & & Reference & & Reference & \\
\hline T2 & $0.92(0.81,1.03)$ & .15 & $1.07(0.95,1.21)$ & .29 & $0.77(0.64,0.92)$ & .005 & $0.96(0.79,1.16)$ & .67 \\
\hline T3 & $1.09(0.98,1.20)$ & .12 & $1.34(1.20,1.51)$ & $<.001$ & $1.21(1.04,1.41)$ & .02 & $1.51(1.27,1.79)$ & $<.001$ \\
\hline $\mathrm{T} 4$ & $2.08(1.76,2.45)$ & $<.001$ & $2.49(2.10,2.96)$ & $<.001$ & $3.36(2.73,4.13)$ & $<.001$ & $3.73(2.99,4.66)$ & $<.001$ \\
\hline \multicolumn{9}{|l|}{ Surgery } \\
\hline No & Reference & & Reference & & Reference & & Reference & \\
\hline Local excision & $0.39(0.33,0.45)$ & $<.001$ & $0.39(0.33,0.46)$ & $<.001$ & $0.26(0.21,0.33)$ & $<.001$ & $0.34(0.27,0.43)$ & $<.001$ \\
\hline
\end{tabular}

$\mathrm{AJCC}=$ American Joint Committee on Cancer system, $\mathrm{Cl}=$ confidence intervals, $\mathrm{HR}=$ hazard ratio *Univariable Cox regression analysis.

$\dagger$ Multivariate analysis adjusted by sex, age, year of diagnosis, race, stage, grade, chemotherapy, radiotherapy, surgery type.

* Included American Indian/AK Native, Asian/Pacific Islander.

marriage on OS for RC disappeared in the subgroup of age over 85 years. Although RCSS was similar between married group and unmarried group, significant difference existed among subsets with year of diagnosis at 2009 to 2010 , aged at 75 to 79 years and patients without surgery, respectively.

Sociodemographic factors affect outcome in a multitude of health conditions. ${ }^{[33-35]}$ Previous studies supported that encourage marriage as mainly source of social support could protect some diseases including cardiovascular disease, ${ }^{[36]}$ head and neck cancer, ${ }^{[1]}$ extremity soft tissue sarcoma, ${ }^{[37]}$ esophageal cancer, ${ }^{[38]}$ prostate cancer. ${ }^{[39]}$ Mechanisms that could explain our current findings can be broadly categorized into effects of social support on adherence and effects of social support arising from reduction of stress. Adherence is the degree to which a patient correctly follows medical advice, and has been considered as an important factor that influences treatment effect and outcome of patients. Poor outcome and diagnosed with cancer result in depression which generate poor adherence to painful therapy.
Mates can be spiritual pillars and relieve patients' depression or discontinuation of medications. ${ }^{[40-43]}$ On the one hand, partners urge patients to screen health status and advocate receiving aggressive treatments; ${ }^{[36,44]}$ on the other hand, marital status provide hope for patients. Married patients experienced better adherence than unmarried, such as immediately seeking for medical attending and receive chemotherapy or radiotherapy. ${ }^{[45,46]}$ Evidence also found that married cancer patients were diagnosed with earlier stage than those were unmarried ${ }^{[12,27,47]}$ and were more likely to receive recommended treatments. ${ }^{[47,48]}$ Furthermore, researches revealed that social support can reduce stress responses, ${ }^{[49,50]}$ for example, the effect of social support on attenuated risk of cardiovascular events and down-regulating cortisol reactivity to mental stress were supported by laboratory settings. ${ }^{[51-53]}$ Marital status not only reduces stress response, but also eases the financial strain. Medical expenses will exert a huge pressure to a family, however, another income from a spouse can reduce the financial strain and weaken anxiety. ${ }^{[54,55]}$ 
Aizer et $\mathrm{al}^{[9]}$ found significant cancer-specific survival benefit of marital status on several cancers including prostate, breast, colorectal, esophageal, and head/neck cancers. They included patients older than 18 years and defined outcome as colorectal cancer specific survival, while we studied RC patients older than 65 years. However, it is more likely for old people to take overall death with many underlying diseases, which is consistent with our findings. The included married patients were 2.5 years younger than unmarried patients on average and were more likely to be both male and white in the early study ${ }^{[9]}$ and married patients were less likely to present with advanced tumor and nodal stage than the unmarried group, in contrast, we had matched the baseline to balance and comparable. Given those, the previous findings are insufficient to apply to the current topic.

Interestingly, in the subgroup of age over 85 years, survival benefit of married on OS disappeared. Reasons for this phenomenon have not found out, however, senility may contribute to that. Contrast with function decline and vestigial organs, effect of social support take a minor place. In addition, we found poorer survival of nonwhite than white people, which consistent with previous studies. ${ }^{[56-58]}$ Weakness of screening, ${ }^{[57]}$ different treating and less aggressive for sequelae treatments, ${ }^{[59]}$ as well as poorer response for chemotherapy ${ }^{[60]}$ for nonwhite population may contribute to that. In addition, we found independent effect of chemotherapy on RCSS, however, the result flipped in multivariate analysis. Given the comparable baseline of this study, further studies are needed to explore reasons contributing on this interesting result.

Our study also has some limitations. Firstly, we cannot randomize our included patients due to the retrospective property. Secondly, the causality cannot be confirmed in this study as an observational study. Thirdly, we conducted propensity score matching to balance the baseline of included patients, at the same time, we lost some samples, and might cause selection bias. In addition, we cannot obtain some essential data such as income, insurance status, residence (rural or urban) and education which related to social support, as well as some detailed data on psychiatric illness, other malignancy, estrogen use in females which may relevant to OS. Although our study does not include extensive socioeconomic factors, the findings indicate a degree of implications. For example, unmarried status indicates a signal of poor social support, medical workers should pay special attention to unmarried groups and provide social support for them. Previous studies reported that we can decrease depression and improve adherence of patients through group interventions, ${ }^{[61]}$ and community skill as well as empathy skill can improve adherence, ${ }^{[62]}$ which could improve outcome of patients.

\section{Conclusions}

Among the aged population, unmarried rectal cancer patients have higher risk of overall death than married patients, except for those older than 85 years. Marital status might have no significant association regarding to RCSS among old people. Further studies could explore what factors could influence RCSS among the aged population.

\section{Acknowledgments}

The authors would like to thank the SEER program for open access to its database.

\section{Author contributions}

Data curation: Zhuyue Li, Kang Wang.

Formal analysis: Zhuyue Li.

Methodology: Kang Wang.

Supervision: Xuemei Zhang, Jin Wen.

Writing - original draft: Zhuyue Li.

Writing - review \& editing: Jin Wen.

\section{References}

[1] Siegel R, Miller K, Fedewa S, et al. Colorectal cancer statistics, 2017. CA Cancer J Clin 2017;67:177-93.

[2] Millino C, Maretto I, Pacchioni B, et al. Gene and microRNA expression are predictive of tumor response in rectal adenocarcinoma patients treated with preoperative chemoradiotherapy. J Cell Physiol 2017;232:426-35.

[3] Santos MD, Silva C, Rocha A, et al. Predictive response value of pre- and postchemoradiotherapy variables in rectal cancer: an analysis of histological data. Pathol Res Int 2016;2016:2164609.

[4] Shan JL, Li Q, He ZX, et al. A population-based study elicits a reverse correlation between age and overall survival in elderly patients with rectal carcinoma receiving adjuvant chemotherapy. Clin Exp Pharmacol Physiol 2015;42:752-65.

[5] Zhang J, Gan L, Wu Z, et al. The influence of marital status on the stage at diagnosis, treatment, and survival of adult patients with gastric cancer: a population-based study. Oncotarget 2017;8:22385-405.

[6] Martinez ME, Anderson K, Murphy JD, et al. Differences in marital status and mortality by race/ethnicity and nativity among California cancer patients. Cancer 2016;122:1570-8.

[7] Jin JJ, Wang W, Dai FX, et al. Marital status and survival in patients with gastric cancer. Cancer Med 2016;5:1821-9.

[8] Mahdi H, Kumar S, Munkarah AR, et al. Prognostic impact of marital status on survival of women with epithelial ovarian cancer. Psychooncology 2013;22:83-8.

[9] Aizer AA, Chen MH, McCarthy EP, et al. Marital status and survival in patients with cancer. J Clin Oncol 2013;31:3869-76.

[10] Baine M, Sahak F, Lin C, et al. Marital status and survival in pancreatic cancer patients: a SEER based analysis. PLoS One 2011;6:e21052.

[11] Inverso G, Mahal BA, Aizer AA, et al. Marital status and head and neck cancer outcomes. Cancer 2015;121:1273-8.

[12] Wang L, Wilson SE, Stewart DB, et al. Marital status and colon cancer outcomes in US Surveillance, Epidemiology and End Results registries: does marriage affect cancer survival by gender and stage? Cancer Epidemiol 2011;35:417-22.

[13] Neuman HB, Patil S, Fuzesi S, et al. Impact of a temporary stoma on the quality of life of rectal cancer patients undergoing treatment. Ann Surg Oncol 2011;18:1397-403.

[14] Law WL, Chu KW, Choi HK. Total pelvic exenteration for locally advanced rectal cancer. J Am Coll Surg 2000;190:78-83.

[15] Guren MG, Eriksen MT, Wiig JN, et al. Quality of life and functional outcome following anterior or abdominoperineal resection for rectal cancer. Eur J Surg Oncol 2005;31:735-42.

[16] Engel J, Kerr J, Schlesinger-Raab A, et al. Quality of life in rectal cancer patients: a four-year prospective study. Ann Surg 2003;238:203-13.

[17] Ross L, Abild-Nielsen AG, Thomsen BL, et al. Quality of life of Danish colorectal cancer patients with and without a stoma. Support Care Cancer 2007;15:505-13.

[18] Russo P, Ravindran B, Katz J, et al. Urinary diversion after total pelvic exenteration for rectal cancer. Ann Surg Oncol 1999;6:732-8.

[19] Lopez MJ, Luna-Perez P. Composite pelvic exenteration: is it worthwhile? Ann Surg Oncol 2004;11:27-33.

[20] Lopez MJ, Spratt JS. Exenterative pelvic surgery. J Surg Oncol 1999;72:102-14.

[21] Hafner GH, Herrera L, Petrelli NJ. Morbidity and mortality after pelvic exenteration for colorectal adenocarcinoma. Ann Surg 1992;215:63-7.

[22] Fossa SD, Cvancarova M, Chen L, et al. Adverse prognostic factors for testicular cancer-specific survival: a population-based study of 27,948 patients. J Clin Oncol 2011;29:963-70.

[23] Rendall MS, Weden MM, Favreault MM, et al. The protective effect of marriage for survival: a review and update. Demography 2011;48: 481-506.

[24] Sammon JD, Morgan M, Djahangirian O, et al. Marital status: a genderindependent risk factor for poorer survival after radical cystectomy. BJU Int 2012;110:1301-9. 
[25] Nelles JL, Joseph SA, Konety BR. The impact of marriage on bladder cancer mortality. Urol Oncol 2009;27:263-7.

[26] Lai H, Lai S, Krongrad A, et al. The effect of marital status on survival in late-stage cancer patients: an analysis based on surveillance, epidemiology, and end results (SEER) data, in the United States. Int J Behav Med 1999;6:150-76.

[27] Reyes Ortiz CA, Freeman JL, Kuo YF, et al. The influence of marital status on stage at diagnosis and survival of older persons with melanoma. J Gerontol A Biol Sci Med Sci 2007;62:892-8.

[28] Jatoi A, Novotny P, Cassivi S, et al. Does marital status impact survival and quality of life in patients with non-small cell lung cancer? Observations from the mayo clinic lung cancer cohort. Oncologist 2007;12:1456-63.

[29] Fritz APC, Jack A. International Classification of Diseases for Oncology. World Health Organization, Geneva, Switzerland:2000.

[30] Sturmer T, Rothman KJ, Avorn J, et al. Treatment effects in the presence of unmeasured confounding: dealing with observations in the tails of the propensity score distribution - a simulation study. Am J Epidemiol 2010;172:843-54.

[31] Zhang Z. Propensity score method: a non-parametric technique to reduce model dependence. Ann Transl Med 2017;5:7.

[32] Scrucca L, Santucci A, Aversa F. Regression modeling of competing risk using R: an in depth guide for clinicians. Bone Marrow Transplant 2010;45:1388-95.

[33] Vallgarda S. Addressing individual behaviours and living conditions: four Nordic public health policies. Scand J Public Health 2011;39(6 suppl):6-10

[34] Arntzen A, Nybo Andersen AM. Social determinants for infant mortality in the Nordic countries, 1980-2001. Scand J Public Health 2004;32:381-9.

[35] Jakobsen L, Niemann T, Thorsgaard N, et al. Dimensions of socioeconomic status and clinical outcome after primary percutaneous coronary intervention. Circ Cardiovasc Interv 2012;5:641-8.

[36] Molloy GJ, Stamatakis E, Randall G, et al. Marital status, gender and cardiovascular mortality: behavioural, psychological distress and metabolic explanations. Social Sci Med (1982) 2009;69:223-8.

[37] Alamanda VK, Song Y, Holt GE. Effect of marital status on treatment and survival of extremity soft tissue sarcoma. Ann Oncol 2014;25:725-9.

[38] Zhang QW, Lin XL, Zhang CH, et al. The influence of marital status on the survival of patients with esophageal cancer: a population-based, propensity-matched study. Oncotarget 2017;8:62261-73.

[39] Abdollah F, Sun M, Thuret R, et al. The effect of marital status on stage and survival of prostate cancer patients treated with radical prostatectomy: a population-based study. Cancer Causes Control 2011;22:1085-95.

[40] DiMatteo MR, Lepper HS, Croghan TW. Depression is a risk factor for noncompliance with medical treatment: meta-analysis of the effects of anxiety and depression on patient adherence. Arch Int Med 2000;160:2101-7.

[41] Li BD, Brown WA, Ampil FL, et al. Patient compliance is critical for equivalent clinical outcomes for breast cancer treated by breastconservation therapy. Ann Surg 2000;231:883-9.

[42] McCowan C, Shearer J, Donnan PT, et al. Cohort study examining tamoxifen adherence and its relationship to mortality in women with breast cancer. Brit J Cancer 2008;99:1763-8.
[43] Richardson JL, Shelton DR, Krailo M, et al. The effect of compliance with treatment on survival among patients with hematologic malignancies. J Clin Oncol 1990;8:356-64.

[44] Schone BS, Weinick RM. Health-related behaviors and the benefits of marriage for elderly persons. Gerontologist 1998;38:618-27.

[45] Cohen SD, Sharma T, Acquaviva K, et al. Social support and chronic kidney disease: an update. Adv Chronic Kidney Dis 2007;14:335-44.

[46] DiMatteo MR. Social support and patient adherence to medical treatment: a meta-analysis. Health Psychol 2004;23:207-18.

[47] Chang SM, Barker FG2nd. Marital status, treatment, and survival in patients with glioblastoma multiforme: a population based study. Cancer 2005; 104:1975-84.

[48] Goodwin JS, Hunt WC, Key CR, et al. The effect of marital status on stage, treatment, and survival of cancer patients. JAMA 1987;258: 3125-30.

[49] Uchino BN. Social support and health: a review of physiological processes potentially underlying links to disease outcomes. J Behav Med 2006;29:377-87.

[50] Cohen S. Psychosocial models of the role of social support in the etiology of physical disease. Health Psychol 1988;7:269-97.

[51] Ditzen B, Schmidt S, Strauss B, et al. Adult attachment and social support interact to reduce psychological but not cortisol responses to stress. J Psychosomatic Res 2008;64:479-86.

[52] Heinrichs M, Baumgartner T, Kirschbaum C, et al. Social support and oxytocin interact to suppress cortisol and subjective responses to psychosocial stress. Biol Psychiatry 2003;54:1389-98.

[53] Eisenberger NI, Taylor SE, Gable SL, et al. Neural pathways link social support to attenuated neuroendocrine stress responses. Neuroimage 2007;35:1601-12.

[54] O'Neill CB, Atoria CL, O'Reilly EM, et al. Costs and trends in pancreatic cancer treatment. Cancer 2012;118:5132-9.

[55] Baker MS, Kessler LG, Urban N, et al. Estimating the treatment costs of breast and lung cancer. Med Care 1991;29:40-9.

[56] Nitzkorski JR, Willis AI, Nick D, et al. Association of race and socioeconomic status and outcomes of patients with rectal cancer. Ann Surg Oncol 2013;20:1142-7.

[57] Morris AM, Billingsley KG, Hayanga AJ, et al. Residual treatment disparities after oncology referral for rectal cancer. J Natl Cancer Inst 2008;100:738-44.

[58] Kim J, Artinyan A, Mailey B, et al. An interaction of race and ethnicity with socioeconomic status in rectal cancer outcomes. Ann Surg 2011;253:647-54.

[59] Ball JK, Elixhauser A. Treatment differences between blacks and whites with colorectal cancer. Med Care 1996;34:970-84.

[60] Dimou A, Syrigos KN, Saif MW. Disparities in colorectal cancer in African-Americans vs Whites: before and after diagnosis. World J Gastroenterol 2009;15:3734-43.

[61] Kissane DW, Grabsch B, Clarke DM, et al. Supportive-expressive group therapy for women with metastatic breast cancer: survival and psychosocial outcome from a randomized controlled trial. Psychooncology 2007;16:277-86.

[62] Kissane DW, Bylund CL, Banerjee SC, et al. Communication skills training for oncology professionals. J Clin Oncol 2012;30:1242-7. 